\title{
Exposure to hypergravity increases serum interleukin-5 and pulmonary infiltration in mice with allergic asthma
}

\author{
TAE YOUNG JANG ${ }^{1}$, KYU-SUNG KIM ${ }^{1}$, CHANG-SHIN PARK ${ }^{1}$, JEONGKU LIM², \\ KANG-CHEOL HUH ${ }^{2}$, MIN-JEONG HEO ${ }^{l}$, AH-YEOUN JUNG ${ }^{l}$, YOUNG HYO KIM ${ }^{l}$ \\ Inha University College of Medicine, Incheon, South Korea \\ ${ }^{2}$ Aerospace Medical Center, South Korea
}

\begin{abstract}
Objective: We evaluated the effect of acute hypergravity $(H G)$ on the immune response in a murine model of allergic asthma.

Material and methods: Twenty-eight BALB/c mice were used. Group A (control group, $n=7$ ) mice were sensitized and challenged with normal saline. Group B (control HG exposure group, $n=7$ ) mice were sensitized, challenged with saline, and exposed to acute $H G(+10 \mathrm{Gz})$ for 4 hours. Group $C$ (asthma group, $n=7$ ) mice were challenged with intraperitoneal and intranasal ovalbumin $(O V A)$ to induce asthma. Group D (asthma HG exposure group, $n=7$ ) mice were exposed to HG for 4 hours after the induction of asthma. We estimated the total and OVA-specific serum IgE, serum titers of various cytokines, and the number of eosinophils, neutrophils, and lymphocytes in bronchoalveolar lavage (BAL) fluid. Histopathology of the lung was also evaluated.

Results: The serum level of interleukin (IL)-5 was significantly higher in Group D $(12.9 \pm 4.9 \mathrm{pg} / \mathrm{ml})$ compared to that in Group C $(4.7 \pm 6.5 \mathrm{pg} / \mathrm{ml}, \mathrm{p}=0.017)$. In BAL fluid, the number of neutrophils was significantly increased in Group D compared to Group $C(p=0.014)$. Group D demonstrated a higher infiltration of inflammatory cells $\left(9973.8 \pm 1642.7 \mathrm{cells} / \mathrm{mm}^{2}\right)$ compared to Group C (7666.3 \pm 586.5 cells $\left./ \mathrm{mm}^{2}, p=0.017\right)$. This tendency of increase in infiltration was not significant in non-asthmatic animals (Group A: $4488.8 \pm 176.1$ cells $/ \mathrm{mm}^{2}$ vs. Group B: $4946.3 \pm 513.7 \mathrm{cells} / \mathrm{mm}^{2}, p>0.05$ ).

Conclusions: Acute $H G$ exacerbated the allergic response by increasing serum IL-5 levels and promoting pulmonary infiltration of inflammatory cells.
\end{abstract}

Key words: cytokines, asthma, allergy, hypergravity.

(Centr Eur J Immunol 2014; 39 (4): 434-440)

\section{Introduction}

During spaceflight, astronauts are exposed to various harmful stimuli such as radiation and acute changes in gravity and barometric pressure [1]. Hypergravity (HG), defined as a gravitational force higher than $+10 \mathrm{Gz}$, during takeoff can critically affect the health of an astronaut. This effect of HG is largely explained by the physiological stress response that provokes the sympathetic nervous system and the hypothalamic-pituitary-adrenal axis [2].

However, few studies have been conducted to investigate the effect of HG on the immune system, and the results of studies conducted thus far are conflicting. Guéguinou et al. reported that serum corticosterone was increased, whereas serum interleukin (IL)-4 and IL-5 levels were unchanged, and IL-10 levels increased marginally after chronic exposure to HG [3]. Galimberti et al. showed that although HG had a negative effect on activation and proliferation of $\mathrm{T}$ cells, it sped up the migration of $\mathrm{T}$ lymphocytes [4]. Other researchers, such as Pecaut et al., reported a decrease in serum interferon $\gamma($ IFN- $\gamma)$ and an increase in IL-4 levels following HG exposure, implying a shift in the Th1/Th2 ratio toward the Th2 immune response and the possible aggravation of allergic disease [5].

Most previous studies on the effect of HG on the immune function used healthy experimental animals. However, to our knowledge, no study has examined the effects of HG on immunological changes in animals with allergic disease or on the clinical course of the disease. Allergic asthma is characterized by bronchoconstriction, airway hyperreactivity, and pulmonary infiltration of inflammatory cells. Therefore, we evaluated the effect of $\mathrm{HG}$ on the immune response in a murine model of asthma by assess-

Correspondence: Prof. Young Hyo Kim, Inha University College of Medicine, Incheon, South Korea, e-mail: inhaorl@inha.ac.kr 
ing: (i) total and ovalbumin (OVA)-specific immunoglobulin $\mathrm{E}(\mathrm{IgE})$ in serum, (ii) serum titers of various cytokines, (iii) the number of different inflammatory cells such as eosinophils, neutrophils, and lymphocytes in the bronchoalveolar lavage (BAL) fluid, and (iv) the histopathology of lung tissue after acute exposure to $+10 \mathrm{Gz}$ HG for 4 hours.

\section{Material and methods}

\section{Animals}

Twenty-eight female BALB/c mice were purchased from Orient Bio (Seongnam, Korea). All mice were 8 to 10 weeks old and free of murine-specific pathogens. They were raised in a controlled environment, with a regular 12-hour light/dark cycle and unrestricted access to food and water. Throughout the experimental period, all mice were fed OVA-free food. All mice used in this study were handled according to a protocol approved by the Institutional Animal Care and Use Committee (INHA 130404202).

\section{Systemic sensitization and intranasal challenge}

Sensitization and intranasal challenge were performed to induce allergic asthma in mice as previously described with minor modifications [6, 7]. Under pathogen-free conditions, OVA (40 $\mu \mathrm{g} / \mathrm{kg}$; Sigma-Aldrich; St. Louis, MO, USA) was diluted in sterile normal saline along with aluminum hydroxide gel (alum adjuvant, $40 \mathrm{mg} / \mathrm{kg}$ ), and intraperitoneally injected to mice on days $1,5,14$, and 21 . Thereafter, mice were challenged intranasally from day 22 through day 35 with a daily dose of OVA diluted in sterile normal saline ( $20 \mu \mathrm{l}$ of $25 \mathrm{mg} / \mathrm{ml}$ OVA per mouse).

\section{Exposure to hypergravity}

We used a centrifuge for the HG experiment. The centrifuge had a $2.0-\mathrm{m}$ arm with a cage at its end to hold the animals. When the arm rotated, an outward force was exerted on the cage. With a rotation speed of $67.0 \mathrm{rpm}$, we produced $\mathrm{HG}$ of $10.0 \mathrm{Gz}$. A high-resolution video camera inside the cage enabled observations of any abnormal behavior of the mice. For control groups without HG exposure, mice were placed in the same room with the HG exposure group, but were not centrifuged (stationary control). Therefore, the experimental environment was essentially the same for all the animals, other than the HG and angular velocity that were applied specifically to the HG exposure group.

Group A (control group, $n=7$ ) mice were given intraperitoneal and intranasal normal saline, without exposure to HG. Mice in group B (control HG exposure group, $n=7$ ) were also challenged with normal saline only, but were then exposed to acute HG for 4 hours, 24 hours after the last intranasal saline challenge. Mice in group C (asthma group, $n=7$ ) received intraperitoneal and intranasal OVA challenge to induce asthma, with no exposure to HG. Finally,
Group D (asthma HG exposure group, $n=7$ ) mice were exposed to HG for 4 hours, 24 hours after the last intranasal OVA challenge for induction of asthma. Immediately after HG exposure, mice were sacrificed, and their serum, BAL fluid, and lung tissues were collected for analysis.

\section{Serum collection and bronchoalveolar lavage fluid harvest}

Serum was collected from the mice by the aortic puncture technique. Bronchoalveolar lavage fluid was harvested by endotracheal lavage with normal saline (approximately $4 \mathrm{ml}$ ) [8]. The BAL fluid was filtered through wet gauze. To determine the viability of the cells and the total cell count, we used the trypan blue exclusion test. The BAL fluid was then centrifuged at $150 \times \mathrm{g}$ for 10 minutes. The pellet was suspended immediately in $4 \mathrm{ml}$ of saline, and total cell numbers were counted in duplicates with a hemocytometer. Subsequently, a 100- $\mu$ l aliquot was centrifuged in a Model 2 Cytospin cytocentrifuge (Shandon Scientific; Pittsburg, PA, USA). Differential cell counts were determined from centrifuged preparations stained with DiffQuick by counting 500 or more cells from each animal at a magnification of $1000 \times$ (oil immersion).

\section{Enzyme-linked immunosorbent assay (ELISA)}

Serum titers of total and OVA-specific IgE were determined by ELISA as previously described [9]. Total IgE was measured and compared with a known mouse IgE standard (BD Pharmingen; San Diego, CA, USA). As there is no mouse OVA-specific IgE standard available, we used the optical density (OD) at $450 \mathrm{~nm}$ instead of concentrations. We also determined serum titers of IL-1, IL-5, IL-10 and IL-13 by the ELISA technique following the manufacturer's instructions.

\section{Histopathology}

Lung tissues were fixed in formalin solution for 3 weeks. They were then embedded in paraffin using standard methods. Sections ( $4 \mu \mathrm{m}$ thick) were stained with hematoxylin and eosin to detect cellular infiltration. The number of infiltrated cells in $1 \mathrm{~mm}^{2}$ of lung parenchyma was counted in 10 random fields of high resolution $(400 \times)$.

\section{Statistical analyses}

We used the Kruskal-Wallis test and Mann-Whitney $U$ test to compare immune responses among the groups, including differences in the total and OVA-specific IgE levels in serum; the number of eosinophils, neutrophils, and lymphocytes in the BAL fluid; the serum titer of each cytokine; and the number of inflammatory cells in lung parenchyma. SPSS 19.0 software (SPSS; Chicago, IL, USA) was used, and $p$-values $<0.05$ were considered statistically significant. 


\section{Results}

Group C (asthma group) and Group D (asthma HG exposure group) mice showed significantly elevated levels of serum total and OVA-specific IgE compared to Group A (control group, $p<0.01$ ). However, no statistically significant differences in the serum titers of total and OVA-specific IgE were observed between Group A and Group B (control HG exposure group) mice. Group D showed slightly higher levels of serum total and OVA-specific IgE compared to Group C, although the difference was not statistically significant $(p>0.05)$ (Fig. 1).

Hypergravity groups (Groups B and D) showed no significant differences in their serum IL-1, IL-10, and IL-13 levels compared to their normogravity counterparts
(Groups A and C; $p>0.05$, Fig. 2A). Groups A and B showed no significant differences in their serum IL-5 levels. However, in asthmatic animals, HG exposure induced a significant increase in IL-5 levels (Group C vs. Group D, $p=0.017$, Fig. 2B).

A higher number of neutrophils and a lower number of lymphocytes were present in the BAL fluid of Group D compared to Group C mice (Fig. 3). In histopathological examinations, Group D demonstrated increased infiltration of inflammatory cells after $\mathrm{HG}$ exposure compared to Group C (Group C: $7666.3 \pm 586.5$ cells $/ \mathrm{mm}^{2}$ vs. Group D: $9973.8 \pm 1642.7$ cells $/ \mathrm{mm}^{2}, p=0.017$, Fig. 4$)$. This tendency of increased infiltration after HG exposure was not statistically significant when non-asthmatic animals were com-
A

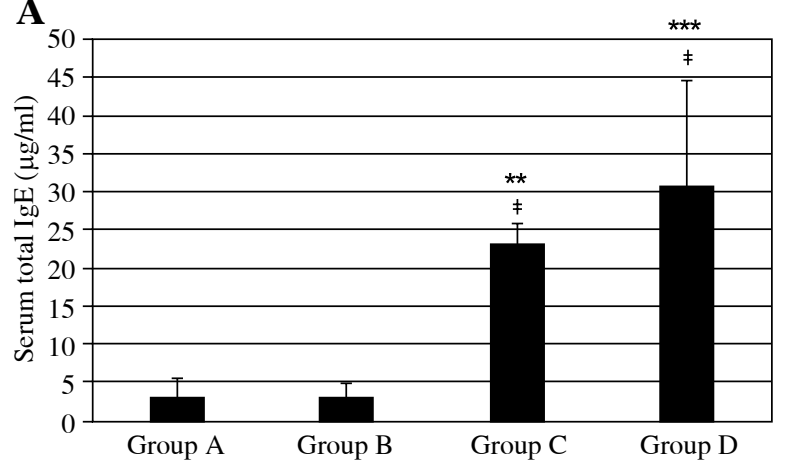

B

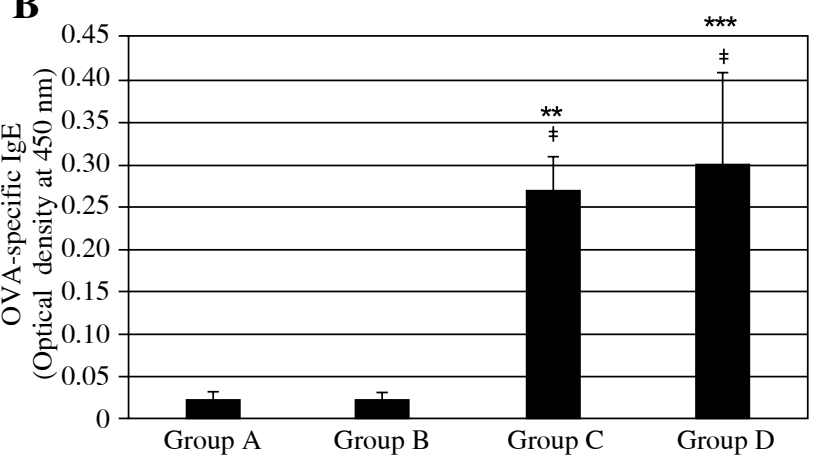

Fig. 1. Serum titers of A) total and B) OVA-specific IgE. Group A (control group), Group B (control hyper-gravity group), Group C (allergic asthma group), group D (asthma hyper-gravity group) (** Significant difference from Group A, $p<0.01, * * *$ Significant difference from Group A, $p<0.001$, $¥$ Significant difference from Group B, $p<0.01$, Kruskal-Wallis test and Mann-Whitney $U$ test)

A

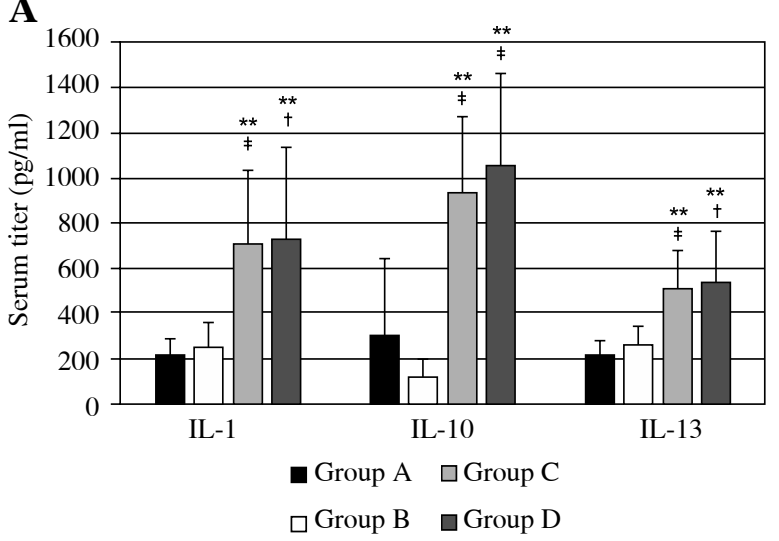

B

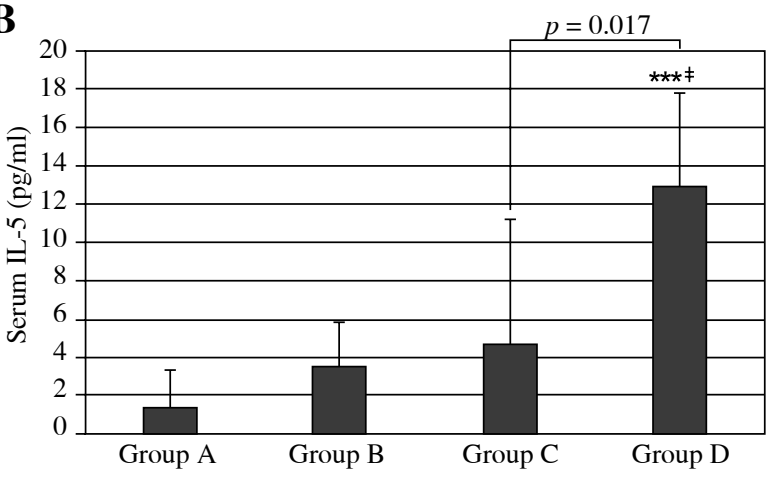

Fig. 2. Serum titers of A) IL-1, IL-10, IL-13, and B) IL-5. Group A (control group), Group B (control hyper-gravity group), Group C (allergic asthma group), group D (asthma hyper-gravity group) (**Significant difference from Group A, $p<0.01, * * *$ Significant difference from Group A, $p<0.001$, $\uparrow$ Significant difference from Group B, $p<0.05$, $¥$ Significant difference from Group B, $p<0.01$, Kruskal-Wallis test and Mann-Whitney $U$ test) 
A

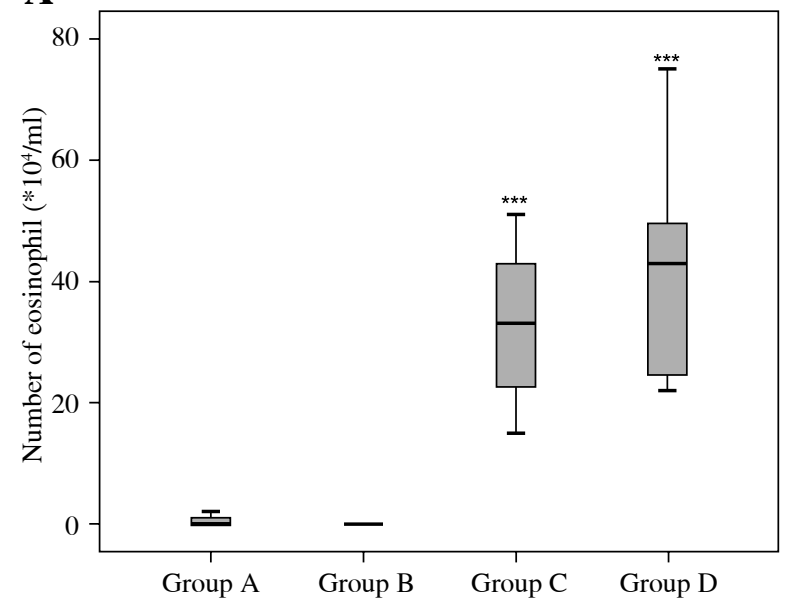

B

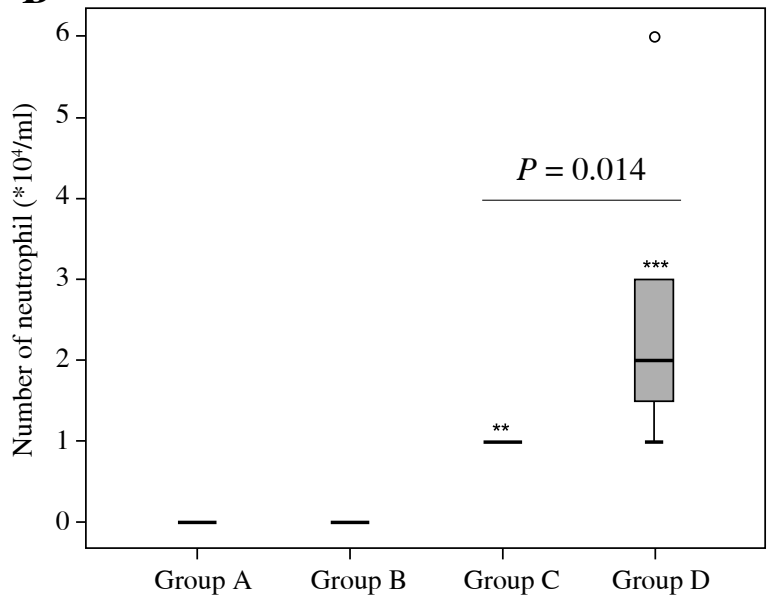

C

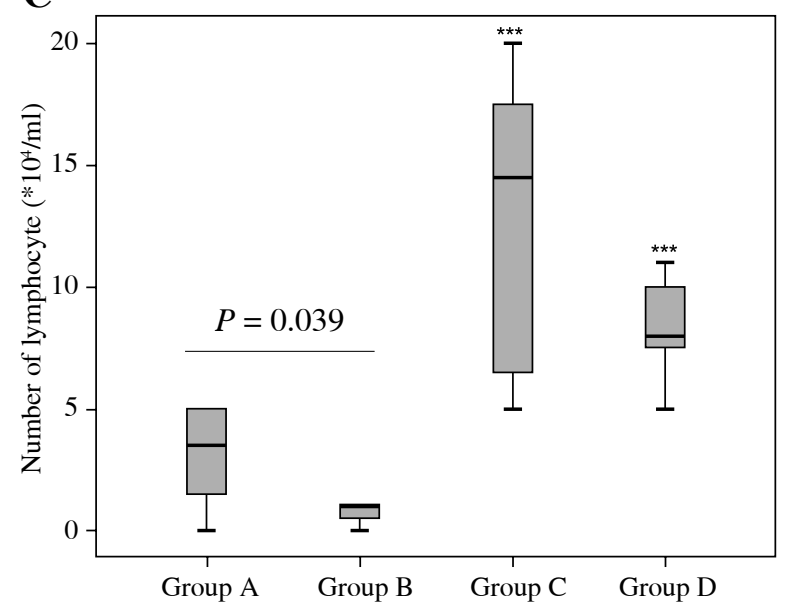

Fig. 3. Number of inflammatory cells in bronchoalveolar lavage fluid. Eosinophils $(\mathbf{A})$, neutrophils $(\mathbf{B})$, and lymphocytes (C) (**Significant difference from Group A, $p<0.01$, ***Significant difference from Group A, $p<0.001$, Kruskal-Wallis test and Mann-Whitney $U$ test)

pared (Group A: $4488.8 \pm 176.1$ cells $/ \mathrm{mm}^{2}$ vs. Group B: $4946.3 \pm 513.7$ cells $/ \mathrm{mm}^{2}, p>0.05$, Fig. 4).

\section{Discussion}

In mice without asthma, serum total and OVA-specific IgE levels did not increase after HG exposure. This result is essentially in agreement with the results of previous studies. Voss reported minor and statistically insignificant fluctuations in serum immunoglobulin levels during a space flight of short duration [10].

In animals with allergic asthma, serum total and OVA-specific IgE levels increased slightly after acute HG exposure. Antonelli et al. reported that the number of mast cells increased after HG exposure, particularly in the blood vessels around the lung airway epithelium [11]. The marginal increase in IgE could result from the degranulation of mast cells that were recruited by the strong gravitational force.
In our study, serum IL-1 levels did not increase after acute HG exposure in non-asthmatic or asthmatic animals. Liu et al. reported that IL-1 expression transiently increased in the rat brain after repeated exposure to $\mathrm{HG}$ of $+14 \mathrm{Gz}$. However, expression levels returned to normal after 48 hours [12]. In our study, IL-1 levels in Group D were not significantly higher than those in the other groups. The minimal change might have been due to the fact that we only used a single exposure to $+10 \mathrm{Gz}$ in our study.

In non-asthmatic mice, there was no statistically significant increase in the serum levels of interleukins involved in IgE isotype switching (IL-13) and in the regulation of eosinophilia (IL-5). These findings are consistent with those of previous studies. Guéguinou et al. reported that chronic $\mathrm{HG}$ ( $+3 \mathrm{Gz}$ for 3 weeks) did not increase serum IL-5 levels in mice [3]. However, we showed that these cytokines increased in asthmatic mice after a single exposure to acute HG. To our knowledge, this is the first study 
A
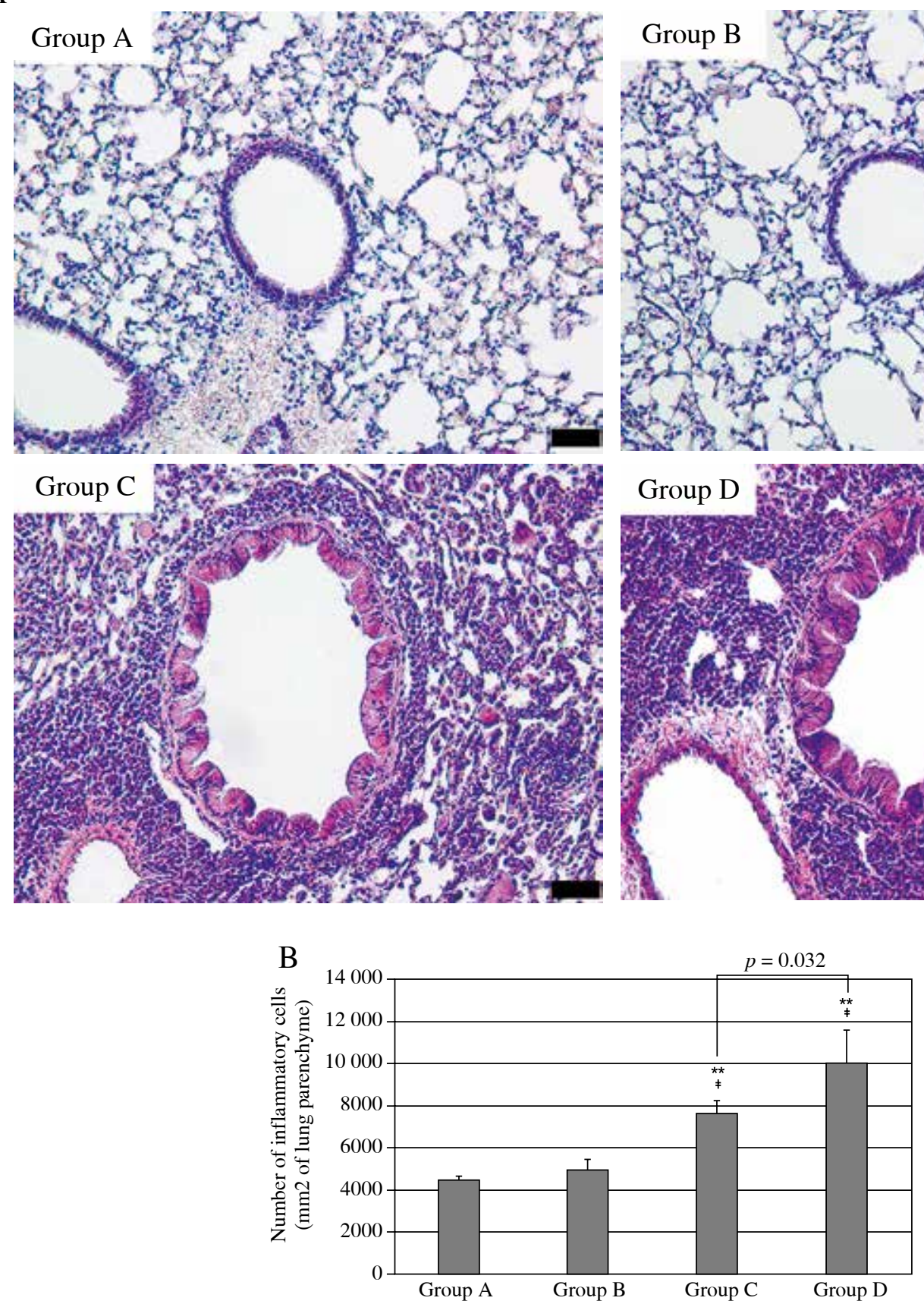

Fig. 4. Histopathologic examination of the lung tissue. A) Hematoxylin and eosin staining, A and B: 200×, C and D: 400×. B) The number of infiltrated inflammatory cells in $1 \mathrm{~mm}^{2}$ of lung parenchyma (Kruskal-Wallis test and Mann-Whitney $U$ test)

to evaluate the effect of acute HG on the humoral immune system in a murine model of allergic asthma. Increased levels of IL-13 could induce an increase in serum IgE, and increased IL-5 could trigger peripheral eosinophilia. Therefore, it is possible that acute HG exposure exacerbates allergic asthma through the induction of the Th2 humoral immune response.
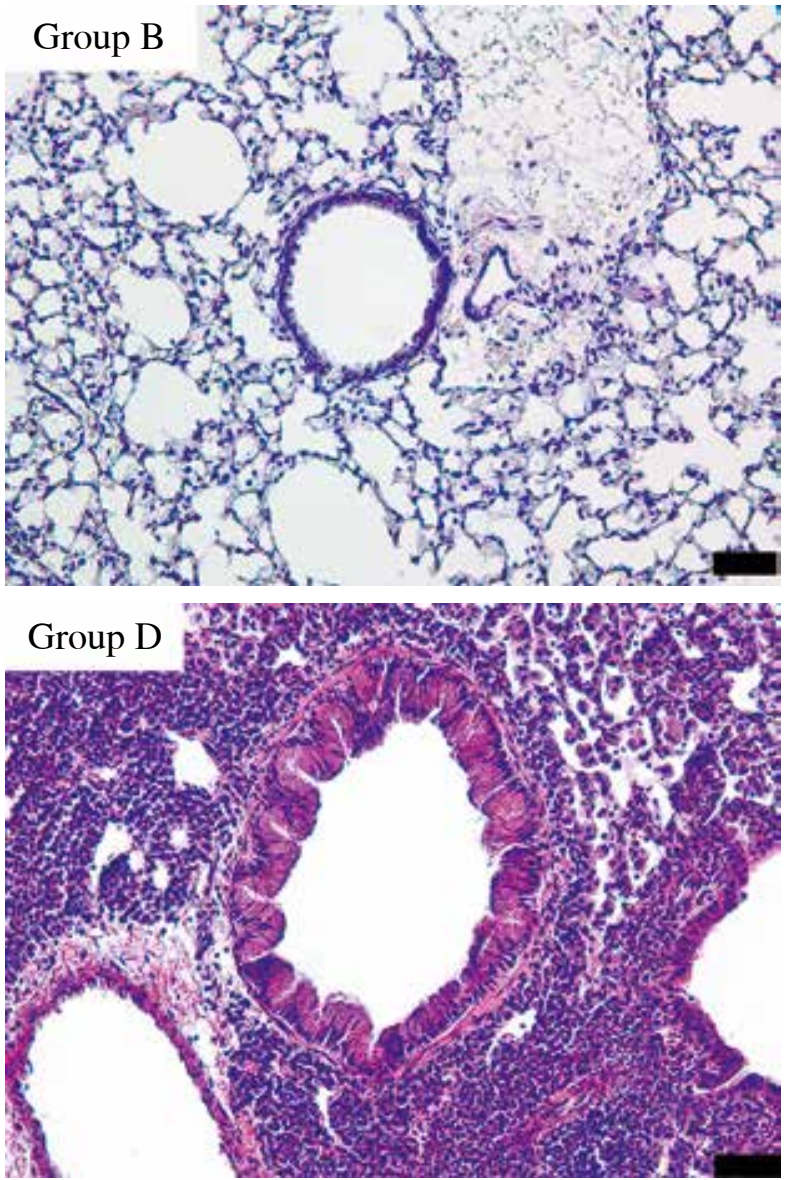
We counted the numbers of eosinophils, neutrophils, and lymphocytes in BAL fluid. To our knowledge, this is the first study to evaluate inflammatory cell numbers in BAL fluid after HG exposure. After HG exposure, the number of inflammatory cells tended to decrease in non-asthmatic animals. Gridley et al. reported that the number of lymphocytes and monocytes (but not granulocytes) decreased after chronic exposure to $+3 \mathrm{Gz}$ for 3 weeks [13]. Therefore, they suggested that progenitors of lymphocytes and monocytes were more affected by HG acceleration than progenitors of granulocytes [13]. In our study, the number of neutrophils increased and the number of lymphocytes decreased when asthmatic mice were exposed to HG. Therefore, increased infiltration of polymorphonuclear cells into the lung could also explain the acute exacerbation of asthma by HG. Furthermore, the degree of lymphocyte depletion after $\mathrm{HG}$ exposure could be a reliable predictor of survival [14]. Although the number of lymphocytes in the BAL fluid of Group D mice was lower than that of Group C mice, the differences were not statistically significant. Therefore, lymphocyte depletion does not appear to be a reliable prognostic factor of survival in asthmatic animals.

By histopathological examination of lung tissue, we confirmed that allergic asthma was exacerbated by acute exposure to $\mathrm{HG}$. Acute exposure to $+10 \mathrm{Gz}$ HG increased the infiltration of inflammatory cells in the lung parenchyma of mice with allergic asthma. To our knowledge, this is the first study to suggest that individuals with allergic asthma are more susceptible to acute changes in gravity. Elucidation of the underlying pathophysiological mechanism will provide further insights into this phenomenon. Cogoli suggested several mechanisms by which acute HG exposure could affect the immune function: (i) activation of signaling pathways related to cellular activation and cytokine secretion, (ii) increase in expression of receptors related to growth factors and immunoregulatory molecules, and (iii) interaction between antigen-presenting cells and lymphocytes [15]. The increase in exhaled nitric oxide and the re-distribution of blood flow to the dependent region of the lung is another possible mechanism $[16,17]$.

A limitation of our study is that we did not include any rotational control group in our experiments, as stressors, rotation, and angular velocity could affect the physiological status of an organism [14]. However, Gridley et al. reported that immunological differences among mice in stationary and rotational control groups were negligible up to 10 days of continuous rotation [13]. Given that we exposed our mice to rotation for only 4 hours, the differences between stationary and rotational control groups would likely be minimal.

In conclusion, in a murine model of allergic asthma, acute exposure to HG exacerbated the allergic disease by increasing serum IL-5 levels and promoting the infiltration of inflammatory cells into the lung parenchyma.
The authors declare no conflict of interest.

This study was supported by the Space Core Technology Development Program through the National Research Foundation of Korea (NRF) funded by the MSIP (Ministry of Science, ICT \& Future Planning) (NRF2013M1A3A3A02042309), the Basic Science Research Program through the National Research Foundation of Korea (NRF) funded by the Ministry of Education, Science, and Technology (NRF-2013R1A1A1006382), and an Inha University Research Grant.

\section{References}

1. Sonnenfeld G, Butel JS, Shearer WT (2003): Effects of the space flight environment on the immune system. Rev Environ Health 18: 1-17.

2. Armario A, Escorihuela RM, Nadal R (2008): Long-term neuroendocrine and behavioural effects of a single exposure to stress in adult animals. Neurosci Biobehav Rev 32: 11211135.

3. Guéguinou N, Bojados M, Jamon M, et al. (2012): Stress response and humoral immune system alterations related to chronic hypergravity in mice. Psychoneuroendocrinology 37 : 137-147.

4. Galimberti M, Tolić-Nřrrelykke IM, Favillini R, et al. (2006): Hypergravity speeds up the development of T-lymphocyte motility. Eur Biophys J 35: 393-400.

5. Pecaut MJ, Miller GM, Nelson GA, Gridley DS (2004): Hypergravity-induced immunomodulation in a rodent model: hematological and lymphocyte function analyses. J Appl Physiol (1985) 97: 29-38.

6. Saito H, Matsumoto K, Denburg AE, et al. (2002): Pathogenesis of murine experimental allergic rhinitis: a study of local and systemic consequences of IL-5 deficiency. J Immunol 168: 3017-3023.

7. Kim YH, Yang TY, Park CS, et al. (2012): Anti-IL-33 antibody has a therapeutic effect in a murine model of allergic rhinitis. Allergy 67: 183-190.

8. Kim YH, Park CS, Lim DH, et al. (2012): Beneficial effect of anti-interleukin-33 on the murine model of allergic inflammation of the lower airway. J Asthma 49: 738-743.

9. El Gazzar M, El Mezayen R, Marecki JC, et al. (2006): Anti-inflammatory effect of thymoquinone in a mouse model of allergic lung inflammation. Int Immunopharmacol 6: 11351142.

10. Voss EW Jr. (1984): Prolonged weightlessness and humoral immunity. Science 225: 214-215.

11. Antonelli A, Santucci D, Amendola T, et al. (2002): Shortterm hypergravity influences NGF and BDNF expression, and mast cell distribution in the lungs and heart of adult male mice. J Gravit Physiol 9: 29-38.

12. Liu HJ, Cai Q, Ji GY, et al. (2000): Changes of mRNA expression of IL-1beta and TNF-alpha in rat brains after repeated exposures to +Gz. Space Med Med Eng (Beijing) 13: 371-373.

13. Gridley DS, Pecaut MJ, Green LM, et al. (2002): Hypergravity-induced immunomodulation in a rodent model: lymphocytes and lymphoid organs. J Gravit Physiol 9: 15-27.

14. Smith AH. Principles of gravitational biology. In: Foundation of Space Biology and Medicine. Calvin M, Gazenko OG 
(eds.). Vol. II, Book 1. Scientific and Technical Information Office, NASA, Washington, DC 1975; 129-162.

15. Cogoli A (1993): The effect of hypogravity and hypergravity on cells of the immune system. J Leukoc Biol 54: 259-268.

16. Karlsson LL, Kerckx Y, Gustafsson LE, et al. (2009): Microgravity decreases and hypergravity increases exhaled nitric oxide. J Appl Physiol (1985) 107: 1431-1437.

17. Petersson J, Rohdin M, Sánchez-Crespo A, et al. (2006): Paradoxical redistribution of pulmonary blood flow in prone and supine humans exposed to hypergravity. J Appl Physiol (1985) 100: 240-248. 